\title{
THE ARITHMETIC, GEOMETRIC AND HARMONIC MEANS IN OPERATOR ALGEBRAS AND TRANSFORMATIONS AMONG THEM
}

\author{
LAJOS MOLNÁR
}

Dedicated to the memory of Professor James Jamison

\begin{abstract}
We study maps on operator algebras which are compatible with, or in another word, respect the most fundamental operator means or pairs of means.
\end{abstract}

\section{INTRODUCTION}

The study of operator means is a very active research area incorporating a number of different directions of research the starting point of which is the beautiful Kubo-Ando theory [12].

Means can also be viewed as operations, and in the papers [15, 16] we determined the corresponding "isomorphisms" for some of the most fundamental means. Namely we described all bijective maps on the space of all positive semidefinite operators on a complex Hilbert space which preserve the geometric, or the arithmetic, or the harmonic mean of operators. Interestingly, the result in [15] led us to the description of Thompson isometries of the positive definite cone of the full operator algebra over a Hilbert space [17]. This latter result provided the main motivation to start systematic investigations concerning so-called generalized MazurUlam theorems whose results appeared in a series of papers from which we cite here only [9] and the survey article [23].

In a certain sense in the present work we extend our former results on mean preservers to the setting of rather general operator algebras.

In what follows, by a $C^{*}$-algebra $\mathcal{A}$ we always mean a unital $C^{*}$-algebra (the unit is usually denoted by $I$ ). The set $\mathcal{A}_{+}^{-1}$ of all positive invertible elements in $\mathcal{A}$ is called the positive definite cone of $\mathcal{A}$. Although there is a way to define means for positive semidefinite operators (not only for positive definite or positive invertible ones), in this paper we consider means only on positive definite cones. In view of the fundamental results of Kubo-Ando theory [12], to make our discussion as simple as possible, we introduce the concept of operator means in the following way. For a given operator monotone function $f:] 0, \infty[\rightarrow \mathbb{R}$ normalized by the condition $f(1)=1$ we define the corresponding mean $\sigma_{f}$ by

$$
A \sigma_{f} B=A^{1 / 2} f\left(A^{-1 / 2} B A^{-1 / 2}\right) A^{1 / 2}, \quad A, B \in \mathcal{A}_{+}^{-1} .
$$

The fundamental properties of $\sigma_{f}$ are the following:

(i) if $A \leq C, B \leq D$, then $A \sigma_{f} B \leq C \sigma_{f} D$;

(ii) for any invertible element $T \in \mathcal{A}$ we have $T\left(A \sigma_{f} B\right) T^{*}=\left(T A T^{*}\right) \sigma_{f}\left(T B T^{*}\right)$

2010 Mathematics Subject Classification. Primary: 47B49, 47A64.

Key words and phrases. Operator means, preservers, $C^{*}$-algebra, von Neumann algebra, positive definite cone.

The author was supported by the "Lendület" Program (LP2012-46/2012) of the Hungarian Academy of Sciences and by the Hungarian Scientific Research Fund (OTKA) Reg. No. K115383. 
for all $A, B, C, D \in \mathcal{A}_{+}^{-1}$. This latter property is usually referred to as the transfer property. A mean is said to be symmetric if it equals the mean obtained by interchanging its variables. For the generating function $f$ this is equivalent to the condition $f(x)=x f\left(x^{-1}\right), x>0$.

The most fundamental symmetric operator means are the arithmetic mean, the geometric mean and the harmonic mean. Their generating operator monotone functions are $x \mapsto(1+x) / 2$, $x \mapsto x^{1 / 2}, x \mapsto(2 x) /(1+x)$, respectively. In what follows we denote the geometric mean by \#.

\section{Results}

In this section we present our results concerning maps between positive definite cones which are sort of morphisms corresponding to means in the sense that they are maps which respect the operation or pairs of operations of the basic means mentioned above. Below we assume that the algebras what we consider are not trivial in the sense that they are not one-dimensional.

We begin with the case of the arithmetic mean. The next result shows that the bijective maps between the positive definite cones of $C^{*}$-algebras that respect the arithmetic mean all originate from Jordan *-isomorphisms of the underlying algebras. A Jordan *-isomorphism between $*$-algebras is a bijective linear map $J$ which respects the square and the *-operations, i.e., which satisfies $J\left(A^{2}\right)=J(A)^{2}$ (or, equivalently, $J(A B+B A)=J(A) J(B)+J(B) J(A)$ ) and $J\left(A^{*}\right)=J(A)^{*}$ for all $A$ (and $B$ ) from its domain. $\mathcal{A}_{s}$.

In what follows, for a $*$-algebra $\mathcal{A}$ we denote the space of all self-adjoint elements in $\mathcal{A}$ by

Proposition 1. Let $\mathcal{A}, \mathcal{B}$ be $C^{*}$-algebras and $\phi: \mathcal{A}_{+}^{-1} \rightarrow \mathcal{B}_{+}^{-1}$ be a bijective map such that

$$
\phi((A+B) / 2)=(\phi(A)+\phi(B)) / 2, \quad A, B \in \mathcal{A}_{+}^{-1} .
$$

Then there is a Jordan ${ }^{*}$-isomorphism $J: \mathcal{A} \rightarrow \mathcal{B}$ and an invertible element $T \in \mathcal{B}$ such that

$$
\phi(A)=T J(A) T^{*}, \quad A \in \mathcal{A}_{+}^{-1} .
$$

Proof. The proof can be given by following the idea of the proof of Lemma 3 in [16] which is based on the use of Kadison's structural result concerning linear order isomorphisms between $C^{*}$-algebras. However, for the sake of completeness we present the argument with all details.

We recall that the equation (1) above is usually called Jensen equation. We learn from the paper [8] that every function from a nonempty $\mathbb{Q}$-convex subset of a linear space $X$ over $\mathbb{Q}$ into another linear space $Y$ over $\mathbb{Q}$ satisfying the Jensen equation can be written in the form $x \mapsto A_{0}+A_{1}(x)$, where $A_{0} \in Y$ and $A_{1}: X \rightarrow Y$ is an additive function. We deduce that there is an element $X \in \mathcal{B}_{s}$ and an additive map $L: \mathcal{A}_{s} \rightarrow \mathcal{B}_{s}$ such that

$$
\phi(A)=L(A)+X, \quad A \in \mathcal{A}_{+}^{-1} .
$$

We assert that $L$ is in fact a continuous linear transformation. First, we know that $L(A) \geq-X$ for every $A \in \mathcal{A}_{+}^{-1}$. It follows that for any element $A \in \mathcal{A}_{s}$ with $\|A\| \leq 1 / 2$ we have $L(I+A) \geq$ $-X$ implying that $L(A) \geq-L(I)-X$. Consequently, there is a negative constant $c \in \mathbb{R}$ such that

$$
L(A) \geq c I
$$

holds whenever $A \in \mathcal{A}_{s},\|A\| \leq 1 / 2$. Inserting $-A$ in the place of $A$, we get $L(-A) \geq c I$ which yields $L(A) \leq-c I$. Therefore, we obtain that

$$
c I \leq L(A) \leq-c I
$$

and hence $\|L(A)\| \leq|c|$ holds for every $A \in \mathcal{A}_{s},\|A\| \leq 1 / 2$. This clearly gives us that the additive map $L$ is continuous and therefore (real-)linear. 
We next prove that $X=0$. Let $A \in \mathcal{A}_{+}^{-1}$ be arbitrary. For every $n \in \mathbb{N}$ we have

$$
n L(A)+X=L(n A)+X=\phi(n A) \geq 0
$$

which gives us that $L(A)+(1 / n) X \geq 0$. If $n$ tends to infinity, we obtain $L(A) \geq 0$. Hence we have $\phi(A)=L(A)+X \geq X$. Since the range of $\phi$ is $\mathcal{B}_{+}^{-1}$, it follows that $0 \geq X$. On the other hand, by the continuity of $L$ we deduce

$$
X=X+L(0)=X+\lim _{n} L((1 / n) I)=\lim _{n} \phi((1 / n) I)
$$

from which it follows that $X \geq 0$. Consequently, we have $X=0$ as asserted.

So, there is a continuous linear transformation $L: \mathcal{A}_{s} \rightarrow \mathcal{B}_{s}$ such that $\phi(A)=L(A), A \in$ $\mathcal{A}_{+}^{-1}$. In the same manner, there corresponds a continuous linear transformation $L^{\prime}: \mathcal{B}_{s} \rightarrow \mathcal{A}_{s}$ to the transformation $\phi^{-1}$. Clearly, we have $L^{\prime}(L(A))=L\left(L^{\prime}(A)\right)=A$ for every $A \in \mathcal{A}_{+}^{-1}$. Since $\mathcal{A}_{+}^{-1}$ linearly generates $\mathcal{A}_{s}$, it follows that $L^{\prime}\left(L(A)=L\left(L^{\prime}(A)\right)=A\right.$ holds for every $A \in \mathcal{A}_{s}$. This shows that the transformation $L$ is bijective and its inverse is $L^{\prime}$. Next, it is easy to see that $L$ is a bijective linear transformation on $\mathcal{A}_{s}$ which preserves the positive elements in both directions, i.e., $A \in \mathcal{A}$ satisfies $A \geq 0$ if and only if $L(A) \geq 0$. Indeed, as $L$ coincides with $\phi$ on $\mathcal{A}_{+}^{-1}$, it sends invertible positive elements to invertible positive elements. Using the continuity of $L$ we obtain that $L$ sends positive elements to positive elements. Applying the same argument for $L^{\prime}$, it then follows that $L$ preserves the positive elements in both directions. Extend $L$ in the obvious manner

$$
A+i B \mapsto L(A)+i L(B), \quad A, B \in \mathcal{A}_{s}
$$

to a bijective linear transformation from $\mathcal{A}$ onto $\mathcal{B}$. We denote this map by the same symbol $L$. Clearly, it is a linear order isomorphism between $\mathcal{A}$ and $\mathcal{B}$. The structure of such maps is wellknown due to an important result of Kadison. By [10, Corollary 5] every bijective unital linear transformation between $C^{*}$-algebras which preserves the positive elements in both directions is necessarily a Jordan ${ }^{*}$-isomorphism. Hence we infer that $L$ is of the form

$$
L(A)=L(I)^{1 / 2} J(A) L(I)^{1 / 2}, \quad A \in \mathcal{A}_{s}
$$

with some Jordan *-isomorphism $J: \mathcal{A} \rightarrow \mathcal{B}$. The proof is complete.

There is a simple correspondence between the arithmetic and harmonic means: for any $A, B \in \mathcal{A}_{+}^{-1}$, the inverse of the arithmetic mean of the inverses of $A, B$ is just the harmonic mean of $A, B$. This yields that the structures of the corresponding preservers are very similar.

Proposition 2. Let $\mathcal{A}, \mathcal{B}$ be $C^{*}$-algebras and $\phi: \mathcal{A}_{+}^{-1} \rightarrow \mathcal{B}_{+}^{-1}$ be a bijective transformation such that

$$
\phi\left(2\left(A^{-1}+B^{-1}\right)^{-1}\right)=2\left(\phi(A)^{-1}+\phi(B)^{-1}\right)^{-1}, \quad A, B \in \mathcal{A}_{+}^{-1} .
$$

Then there is a Jordan ${ }^{*}$-isomorphism $J: \mathcal{A} \rightarrow \mathcal{B}$ and an invertible element $T \in \mathcal{B}$ such that

$$
\phi(A)=T J(A) T^{*}, \quad A \in \mathcal{A}_{+}^{-1} .
$$

Proof. Define $\psi(A)=\phi\left(A^{-1}\right)^{-1}, A \in \mathcal{A}_{+}^{-1}$. Then $\psi$ satisfies the conditions in the previous statement: it is bijective and preserves the arithmetic mean. Recalling that Jordan isomorphisms are compatible with the inverse operation, $J\left(A^{-1}\right)=J(A)^{-1}$ (see, e.g., Proposition 1.3 in [27]), easy application of Proposition 1 gives the required conclusion. 
We continue along this line and next consider the case of the geometric mean which, as we shall see, is definitely more complicated. For example, observe that while in the results above we have not needed the assumption of continuity, in the next result we does need it.

The description of the geometric mean preservers is closely related to the description of so-called Jordan triple isomorphisms. For $C^{*}$-algebras $\mathcal{A}, \mathcal{B}$ we say that the bijective map (no linearity is assumed!) $\psi: \mathcal{A}_{+}^{-1} \rightarrow \mathcal{B}_{+}^{-1}$ is a Jordan triple isomorphism if it satisfies

$$
\psi(A B A)=\psi(A) \psi(B) \psi(A), \quad A, B \in \mathcal{A}_{+}^{-1} .
$$

If bijectivity is not assumed here, we call the map a Jordan triple map. We shall make use the following result which has appeared as Theorem 5 in [23]. A linear functional $l: \mathcal{A} \rightarrow \mathbb{C}$ on an algebra $\mathcal{A}$ is said to be tracial if it has the property $l(A B)=l(B A), A, B \in \mathcal{A}$.

Theorem 3. (23]) Assume $\mathcal{A}, \mathcal{B}$ are von Neumann algebras and $\mathcal{A}$ is a factor not of type $\mathrm{I}_{2}$. Let $\psi: \mathcal{A}_{+}^{-1} \rightarrow \mathcal{B}_{+}^{-1}$ be a continuous Jordan triple isomorphism. Then there is either an algebra ${ }^{*}$-isomorphism or an algebra ${ }^{*}$-antiisomorphism $\theta: \mathcal{A} \rightarrow \mathcal{B}$, a number $c \in\{-1,1\}$, and a continuous tracial linear functional $l: \mathcal{A} \rightarrow \mathbb{C}$ which is real valued on $\mathcal{A}_{s}$ and satisfies $l(I) \neq-c$ such that

$$
\psi(A)=e^{l(\log A)} \theta\left(A^{c}\right), \quad A \in \mathcal{A}_{+}^{-1} .
$$

Conversely, for any algebra ${ }^{*}$-isomorphism or algebra ${ }^{*}$-antiisomorphism $\theta: \mathcal{A} \rightarrow \mathcal{B}$, number $c \in\{-1,1\}$, and continuous tracial linear functional $l: \mathcal{A} \rightarrow \mathbb{C}$ which is real valued on $\mathcal{A}_{s}$ and satisfies $l(I) \neq-c$, the above displayed formula (2) defines a continuous Jordan triple isomorphism between $\mathcal{A}_{+}^{-1}$ and $\mathcal{B}_{+}^{-1}$.

Our result on geometric mean preservers now reads as follows. Recall that on any finite von Neumann algebra there is a unique center-valued positive linear functional which is tracial and acts as the identity on the center, see 8.2.8. Theorem in [11]. This functional is called the normalized trace, and we denote it by $\mathrm{Tr}$.

Theorem 4. Assume $\mathcal{A}, \mathcal{B}$ are von Neumann algebras and $\mathcal{A}$ is a factor. Let $\phi: \mathcal{A}_{+}^{-1} \rightarrow \mathcal{B}_{+}^{-1}$ be a continuous bijective map which satisfies

$$
\phi(A \# B)=\phi(A) \# \phi(B), \quad A, B \in \mathcal{A}_{+}^{-1} .
$$

Suppose $\mathcal{A}$ is of infinite type. Then there is either an algebra *isomorphism or an algebra ${ }^{*}$-antiisomorphism $\theta: \mathcal{A} \rightarrow \mathcal{B}$, a number $c \in\{-1,1\}$, and an invertible element $T \in \mathcal{B}$ such that

$$
\phi(A)=T \theta\left(A^{c}\right) T^{*}, \quad A \in \mathcal{A}_{+}^{-1} .
$$

Assume $\mathcal{A}$ is of finite type. Then there is either an algebra ${ }^{*}$-isomorphism or an algebra ${ }^{*}$ antiisomorphism $\theta: \mathcal{A} \rightarrow \mathcal{B}$, a number $c \in\{-1,1\}$, an invertible element $T \in \mathcal{B}$, and a real number $d$ with $d \neq-c$ such that

$$
\phi(A)=e^{d \operatorname{Tr}(\log A)} T \theta\left(A^{c}\right) T^{*}, \quad A \in \mathcal{A}_{+}^{-1} .
$$

Proof. The geometric mean $A \# B$ is known to be the unique solution $X \in \mathcal{A}_{+}^{-1}$ of the equation $X A^{-1} X=B$. This is called Anderson-Trapp theorem, for the original source see [3]. It follows that $\phi$ satisfies

$$
\phi(X) \phi(A)^{-1} \phi(X)=\phi(B)=\phi\left(X A^{-1} X\right)
$$

for all $A, X \in \mathcal{A}_{+}^{-1}$. Consider the continuous bijective map $\psi: \mathcal{A}_{+}^{-1} \rightarrow \mathcal{B}_{+}^{-1}, \psi()=$. $\phi(I)^{-1 / 2} \phi(.) \phi(I)^{-1 / 2}$. This also clearly satisfies the last equality and it maps the unit to the unit. It follows that $\psi$ fulfills $\psi\left(A^{-1}\right)=\psi(A)^{-1}, A \in \mathcal{A}_{+}^{-1}$, too, and then we have

$$
\psi(A B A)=\psi(A) \psi(B) \psi(A), \quad A, B \in \mathcal{A}_{+}^{-1} .
$$


Consequently, $\psi$ is a continuous Jordan triple isomorphism between $\mathcal{A}_{+}^{-1}$ and $\mathcal{B}_{+}^{-1}$. Assume $\mathcal{A}$ is not of type $\mathrm{I}_{2}$. We then apply the previous result Theorem 3. Furthermore, we use the probably folk result (which was given also in [24, Proposition 2]) that says the following: If $l$ is a nonzero continuous tracial linear functional $l$ on the factor $\mathcal{A}$ (positivity of $l$ is not assumed), then $\mathcal{A}$ is of finite type and $l$ is a scalar multiple of the normalized trace on $\mathcal{A}$. The proof can be completed easily.

Assume now that $\mathcal{A}$ is of type $\mathrm{I}_{2}$. In that case $\mathcal{A}$ is isomorphic to the algebra of all 2 by 2 complex matrices. Lemma 16 in [23] states that any continuous Jordan triple map (a map respecting the product $A B A$ ) between the positive definite cones of $C^{*}$-algebras is of the form $\exp \circ L \circ \log$ where $L$ is a linear map between the self-adjoint parts of the underlying algebras which preserves commutativity. Clearly, in the case of continuous Jordan triple isomorphims this linear map is a linear bijection which preserves commutativity in both directions. It follows that we necessarily have $\mathcal{B}$ is also a type $\mathrm{I}_{2}$ factor. Therefore, we can consider the problem formulated for the positive definite cone of the space of 2 by 2 matrices. The structure of continuous Jordan triple isomorphisms in that case has recently been described in [25]. Theorem 2 in that paper gives exactly what is needed to complete the proof.

Remark 5. Observe that the converse of the statement in Theorem 4 is also valid: any transformation of any of the forms that appear in the statement is a continuous bijective map which respects the geometric mean. This follows essentially using the last part of Theorem 3 and the transfer property of operator means. The converses of the statements in Propositions 1 and 2 are also true, this can be checked trivially.

One can be interested and ask what happens if we omit the bijectivity assumptions in the results above. In that case we have only a partial result and only concerning the geometric mean. Indeed, in the case of the positive definite cone $\mathbb{P}_{n}$ in the algebra of all $n$ by $n$ complex matrices one can obtain a precise structural result for the not necessarily bijective continuous maps which preserve the geometric mean. In fact, that follows from corresponding results on continuous Jordan triple maps, namely from [22, Theorem 1] for the case where $n \geq 3$ and from [25, Theorem 1] for the case where $n=2$. What one needs to do is to relate a Jordan triple map to our geometric mean preserver exactly in the way as we have done in the proof of Theorem 4 above.

As for the same problem concerning the arithmetic mean or the harmonic mean, due to the already mentioned close connection between those two means, the questions are equivalent, so let us consider only the one for the arithmetic mean. Clearly, every unital positive linear map on the algebra of all $n$ by $n$ complex matrices gives rise to a map on $\mathbb{P}_{n}$ preserving the arithmetic mean. But even for such maps no structural result is known. A relating famous result due to Choi says that every so-called completely positive linear maps on the full matrix algebra is of the form $A \mapsto \sum_{k=1}^{m} T_{k} A T_{k}^{*}$, where $T_{k}$ 's are given $n$ by $n$ matrices. (These maps are widely used in quantum information theory, see the concept of quantum operations.)

In the next section of the paper we consider transformations between different means. In view of our previous results and the correspondence between the arithmetic and harmonic means, for that latter pair of means the problem is not interesting, or better say it is trivial. Concerning the geometric mean and the arithmetic mean (or, equivalently, the harmonic mean), the problem is rather algebraic in nature. Namely, we show below that there can be an injective transformation which transforms the geometric mean to the arithmetic mean only in the case of commutative algebras. To see this, we first recall the following algebraic concepts related to associativity.

Concerning any operation $(a, b) \mapsto a \diamond b$ on a set $X$ consider the following weak associativity conditions (for all $a, b \in X)$ :

(a) $(a \diamond a) \diamond b=a \diamond(a \diamond b)$ which is called left alternative identity; 
(b) $(a \diamond b) \diamond b=a \diamond(b \diamond b)$ which is called right alternative identity;

(c) $a \diamond(b \diamond a)=(a \diamond b) \diamond a$ which is called flexible identity.

In the case of the positive definite cone of a $C^{*}$-algebra $\mathcal{A}$, for the standard K-loop product $A \sqcup B=A^{1 / 2} B A^{1 / 2}, A, B \in \mathcal{A}_{+}^{-1}$ these identities were investigated in the paper [4].

Let us now consider the operation $A \star B=(A \# B)^{2}, A, B \in \mathcal{A}_{+}^{-1}$. The geometric mean is symmetric, it satisfies $A \# B=B \# A, A, B \in \mathcal{A}_{+}^{-1}$. This implies that $\star$ has the flexible identity and for the same reason the left and right alternative identities are equivalent. Concerning these identities we have the following result.

Proposition 6. Let $\mathcal{A}$ be a $C^{*}$-algebra. The operation $A \star B=(A \# B)^{2}, A, B \in \mathcal{A}_{+}^{-1}$ satisfies the left (or the right) alternative identity if and only if the algebra $\mathcal{A}$ is commutative.

Proof. If $\mathcal{A}$ is commutative, then $A \star B=A B$ and there is nothing to prove. Assume $\star$ satisfies the left alternative identity. Then we have

$$
A^{2} \# B=A \#(A \# B)^{2}, \quad A, B \in \mathcal{A}_{+}^{-1} .
$$

This implies

$$
A\left(A^{-1} B A^{-1}\right)^{1 / 2} A=A^{1 / 2}\left(\left(A^{-1 / 2} B A^{-1 / 2}\right)^{1 / 2} A\left(A^{-1 / 2} B A^{-1 / 2}\right)^{1 / 2}\right)^{1 / 2} A^{1 / 2} .
$$

Multiplying both sides by $A^{-1 / 2}$ we have

$$
A^{1 / 2}\left(A^{-1} B A^{-1}\right)^{1 / 2} A^{1 / 2}=\left|A^{1 / 2}\left(A^{-1 / 2} B A^{-1 / 2}\right)^{1 / 2}\right|
$$

(here $|C|=\left(C^{*} C\right)^{1 / 2}$ ). Setting $X=A^{1 / 2}, Y^{2}=A^{-1} B A^{-1}$ we deduce

$$
X Y X=\left|X\left(X Y^{2} X\right)^{1 / 2}\right|
$$

Taking square we obtain

$$
X Y X^{2} Y X=\left(X Y^{2} X\right)^{1 / 2} X^{2}\left(X Y^{2} X\right)^{1 / 2}
$$

For the above mentioned standard K-loop product $\square$ this gives that

$$
X^{2} \boxminus\left(Y^{2} \boxminus X^{2}\right)=\left(X^{2} \boxminus Y^{2}\right) \boxminus X^{2}
$$

holds for all $X, Y \in \mathcal{A}_{+}^{-1}$. This means that $\square$ satisfies the flexible identity. It was proved in $[4$, Proposition 3] that it holds if and only if $\mathcal{A}$ is commutative. The proof is complete.

We remark that similar commutativity results have been obtained in [1] concerning so-called gyrogroup structures on the positive definite cones of $C^{*}$-algebras.

Using the previous statement we can show in large generality that in non-commutative algebras there is no injective map that would transform the geometric mean to the algebraic mean. The precise result reads as follows (we note that similar result for uniquely 2-divisible groups was obtained in [21]).

Proposition 7. Assume $\mathcal{A}$ is a $C^{*}$-algebra and $\mathcal{S}$ is a left cancellative semigroup. Let $\phi$ : $\mathcal{A}_{+}^{-1} \rightarrow \mathcal{S}$ be an injective map which satisfies

$$
\phi(A \# B)^{2}=\phi(A) \phi(B), \quad A, B \in \mathcal{A}_{+}^{-1} .
$$

Then $\mathcal{A}$ is necessarily commutative. 
Proof. We have $\phi\left(A^{1 / 2}\right)^{2}=\phi(I) \phi(A)$ and $\phi\left(A^{1 / 2}\right)^{2}=\phi(A) \phi(I), A \in \mathcal{A}_{+}^{-1}$. It follows that $\phi(I)$ commutes with the range of $\phi$ and we also obtain $\phi(A)^{2}=\phi(I) \phi\left(A^{2}\right), A \in \mathcal{A}_{+}^{-1}$. We have

$$
\phi(A) \phi(B)=\phi(A \# B)^{2}=\phi(I) \phi\left((A \# B)^{2}\right)=\phi(I) \phi(A \star B), \quad A, B \in \mathcal{A}_{+}^{-1} .
$$

We now compute on the one hand

$$
(\phi(A) \phi(B)) \phi(C)=(\phi(I) \phi(A \star B)) \phi(C)=\phi(I)^{2} \phi((A \star B) \star C)
$$

and on the other hand

$$
\phi(A)(\phi(B) \phi(C)))=\phi(A)(\phi(I) \phi(B \star C))=\phi(I)(\phi(A) \phi(B \star C))=\phi(I)^{2} \phi(A \star(B \star C)) .
$$

By the left cancellation property of $\mathcal{S}$ and the injectivity of $\phi$ it follows that

$$
(A \star B) \star C=A \star(B \star C), \quad A, B, C \in \mathcal{A}_{+}^{-1}
$$

which means that the $\star$ operation is associative. Applying Proposition 6 it follows that $\mathcal{A}$ is commutative.

We remark that if $\mathcal{A}$ is a commutative $C^{*}$-algebra and $\mathcal{S}$ is the additive group of all selfadjoint elements in $\mathcal{A}$, then the map $A \mapsto \log A$ is clearly a bijective map from $\mathcal{A}_{+}^{-1}$ onto $\mathcal{S}$ which satisfies (5).

What about not necessarily injective maps? In the next result we determine the structure of all continuous maps from the positive definite cone of a von Neumann factor to a Banach space which transforms the geometric mean to the arithmetic mean. In the proof we shall use the deep and famous result known as the solution of the Mackey-Gleason problem which concerns measures on projection lattices in von Neumann algebras. A version of the Lie-Trotter product formula is also applied.

Theorem 8. Let $\mathcal{A}$ be a von Neumann factor, $X$ be a Banach space. Let $\phi: \mathcal{A}_{+}^{-1} \rightarrow X$ be a continuous map such that

$$
\phi(A \# B)=(1 / 2)(\phi(A)+\phi(B)) \quad A, B \in \mathcal{A}_{+}^{-1} .
$$

If $\mathcal{A}$ is infinite, then $\phi$ is necessarily constant. If $\mathcal{A}$ is of finite type, then there are vectors $x_{0}, y_{0} \in X$ such that $\phi$ is of the form

$$
\phi(A)=\operatorname{Tr}(\log A) \cdot x_{0}+y_{0}, \quad A \in \mathcal{A}_{+}^{-1} .
$$

Proof. Assume that $\phi$ is not constant. Clearly, considering the map $A \rightarrow \phi(A)-\phi(I)$ we may and hence we do assume that $\phi(I)=0$ and $\phi$ is not identically zero.

We now follow the idea in the proof of Theorem 4. First recall that for any $A, B \in \mathcal{A}_{+}^{-1}$, the element $X=A \# B$ is the unique solution of the equation $X A^{-1} X=B$ in $\mathcal{A}_{+}^{-1}$. Therefore, we can write

$$
\phi(X)=(1 / 2)\left(\phi(A)+\phi\left(X A^{-1} X\right)\right), \quad X, A \in \mathcal{A}_{+}^{-1} .
$$

This implies

$$
2 \phi(X)-\phi(A)=\phi\left(X A^{-1} X\right), \quad X, A \in \mathcal{A}_{+}^{-1}
$$

and inserting $X=I$ we deduce $\phi(A)=-\phi\left(A^{-1}\right)$. Consequently, we obtain

$$
2 \phi(X)+\phi(A)=\phi(X A X), \quad X, A \in \mathcal{A}_{+}^{-1},
$$

or, using different symbols,

$$
\phi(A B A)=2 \phi(A)+\phi(B), \quad A, B \in \mathcal{A}_{+}^{-1} .
$$


It follows that $\phi\left(A^{n}\right)=n \phi(A)$ holds for all integers $n$ and then that $\phi\left(A^{r}\right)=r \phi(A)$ holds also for all rational numbers $r$. Apparently, we have

$$
\phi\left(A^{1 / 2} B A^{1 / 2}\right)=\phi(A)+\phi(B), \quad A, B \in \mathcal{A}_{+}^{-1} .
$$

It is assumed that $\phi$ is continuous. This implies that we have some real number $0<\delta<1$ such that for any $X \in \mathcal{A}_{+}^{-1}$ with $(1-\delta) I \leq X \leq(1+\delta) I$ it follows that $\|\phi(X)\| \leq 1$. Applying the last observation in the previous paragraph by taking high enough roots we can deduce that there is a number $\epsilon>0$ such that for any $X \in \mathcal{A}_{+}^{-1}$ with $I \leq X \leq 3 I$ we have $\|\phi(X)\| \leq \epsilon$. In particular, the map $P \rightarrow \phi\left(e^{P}\right)$ on the lattice $\mathcal{P}(\mathcal{A})$ of all projections in $\mathcal{A}$ is bounded. Moreover, by $(8)$, for any $P, Q \in \mathcal{P}(\mathcal{A})$ with $P Q=0$ we have that $P, Q$ commute and then that

$$
\phi\left(e^{P+Q}\right)=\phi\left(e^{P / 2} e^{Q} e^{P / 2}\right)=\phi\left(e^{P}\right)+\phi\left(e^{Q}\right) .
$$

This implies that $P \rightarrow \phi\left(e^{P}\right)$ is a bounded finitely additive $X$-valued measure on $\mathcal{P}(\mathcal{A})$. By the solution of the Mackey-Gleason problem due to Bunce and Wright [6], if $\mathcal{A}$ is not of type $\mathrm{I}_{2}$, then it follows that there is a bounded linear operator $L: \mathcal{A} \rightarrow X$ such that

$$
\phi\left(e^{P}\right)=L(P), \quad P \in \mathcal{P}(\mathcal{A}) .
$$

Since for commuting $A, B \in \mathcal{A}_{+}^{-1}$ we clearly have $\phi(A B)=\phi(A)+\phi(B)$, it follows easily from the spectral theorem and from the continuity of $\phi$ that we have $\phi\left(e^{S}\right)=L(S)$ for every self-adjoint element $S \in \mathcal{A}_{s}$. This means that

$$
\phi(A)=L(\log A), \quad A \in \mathcal{A}_{+}^{-1} .
$$

Using (7), by the linearity of $L$ we obtain

$$
\log A B A-2 \log A-\log B \in \operatorname{ker} L
$$

We now apply one of our recent results. Theorem 1 in [24] states that the closed linear span of the set

$$
\left\{\log A B A-2 \log A-\log B: A, B \in \mathcal{A}_{+}^{-1}\right\}
$$

is either the whole algebra $\mathcal{A}$ meaning that $L, \phi$ are identically zero, or this closed linear span is a proper subspace of $\mathcal{A}$ in which case $\mathcal{A}$ is a finite factor and that span coincides with the kernel of the unique normalized trace functional on $\mathcal{A}$. Therefore, if $\phi$ is not identically zero, then so is $L$ and it follows that ker $L$ equals the kernel of Tr. We now easily obtain that for some vector $x_{0} \in X$ we have

$$
\phi(A)=L(\log A)=\operatorname{Tr}(\log A) \cdot x_{0}, \quad A \in \mathcal{A}_{+}^{-1} .
$$

This finishes the proof if $\mathcal{A}$ is not of type $\mathrm{I}_{2}$.

Let us next consider the case where $\mathcal{A}$ is of type $\mathrm{I}_{2}$, i.e., $\mathcal{A}$ is isometrically isomorphic to the algebra of all 2 by 2 complex matrices. Pick self-adjoint elements $S, T \in \mathcal{A}_{s}$. Inserting $e^{S / n}, e^{T / n}$ in the places of $A$ and $B$, respectively, in (8) we have

$$
\begin{gathered}
\phi\left(\left(e^{S / 2 n} e^{T / n} e^{S / 2 n}\right)^{n}\right)=n\left(\phi\left(e^{S / n}\right)+\phi\left(e^{T / n}\right)\right) \\
=n\left(\phi\left(e^{S}\right) / n+\phi\left(e^{T}\right) / n\right)=\phi\left(e^{S}\right)+\phi\left(e^{T}\right)
\end{gathered}
$$

for every $n$. By a version of Lie-Trotter product formula (see, e.g., [5], Exercise IX.1.4. on page 255) we have

$$
\lim _{n \rightarrow \infty}\left(e^{S / 2 n} e^{T / n} e^{S / 2 n}\right)^{n}=e^{S+T}
$$


where the convergence holds in the norm topology (we remark that similar formula holds in infinite dimensions too, but there the limit should be taken in the strong operator topology). We obtain

$$
\phi\left(e^{S+T}\right)=\phi\left(e^{S}\right)+\phi\left(e^{T}\right)
$$

for all self-adjoint elements $S, T \in \mathcal{A}_{s}$. It follows that the map

$$
S \mapsto \phi\left(e^{S}\right)
$$

is a continuous additive map on $\mathcal{A}_{s}$. Therefore, it is linear and hence it has an extension to a (continuous) linear operator $L: \mathcal{A} \rightarrow X$ with the property that

$$
\phi(A)=L(\log A), \quad A \in \mathcal{A}_{+}^{-1} .
$$

The proof can be completed just as in the case of not type $I_{2}$ factors.

Remark 9. We make a few remarks concerning the above result.

Observe first that the above theorem can also be viewed as a characterization of finite von Neumann factors together with the trace functional and the logarithmic function. In fact, for example, one can formulate the following assertion: If $\mathcal{A}$ is a von Neumann factor, $l$ is a nonzero continuous (real-)linear functional on $\mathcal{A}_{s}$ and $f$ is a nonconstant continuous real function on the positive real numbers such that

$$
l(f(A \# B))=(1 / 2)(l(f(A))+l(f(B))), \quad A, B \in \mathcal{A}_{+}^{-1},
$$

i.e., the transformation $l \circ f$ sends the geometric mean to the arithmetic mean, then the algebra $\mathcal{A}$ is necessarily of finite type, $l$ is a scalar multiple of the unique normalized trace on $\mathcal{A}$ and $f$ is an affine function of the logarithmic function (cf. [24, Corollary 3]).

Next observe that by the appearance of the logarithmic function in the form (6) above, the range of the transformation $\phi$ is either a 0 - or a 1-dimensional linear manifold. It follows that there is simply no non-constant continuous map between the positive definite cones of von Neumann factors which would transform the geometric mean to the arithmetic mean.

On the other hand, one would ask about transforming the arithmetic mean to the geometric mean. Regarding this question we have only a particular and finite dimensional result for which we refer to [19, Theorem 2].

The argument used in the proof of the previous theorem can be applied to give a characterization of the so-called logarithmic product on the positive definite cone. The logarithmic product of $A, B \in \mathcal{A}_{+}^{-1}$ is $\exp (\log A+\log B)$. This concept originally emerged from computational geometry [2] but soon after serious applications have been found regarding the differential geometry of spaces of positive definite operators which is a large and active area of research in present days. Clearly, the logarithmic product makes the positive definite cone a commutative group. Characterization of this product by the property that it is an ordered commutative group operation with respect to the chaotic order was given in [18, Theorem 8] for the full operator algebra over a Hilbert space. Our new characterization result reads as follows.

Theorem 10. Let $\mathcal{A}$ be a von Neumann algebra without a type $I_{2}$ direct summand and $X$ be a Banach space. Let $\bullet$ be a group operation on $\mathcal{A}_{+}^{-1}$ which coincides with the original product on commuting elements in $\mathcal{A}_{+}^{-1}$ and assume that there is an injective continuous map $\phi: \mathcal{A}_{+}^{-1} \rightarrow X$ such that

$$
\phi(A \bullet B)=\phi(A)+\phi(B), \quad A, B \in \mathcal{A}_{+}^{-1} .
$$

Then we have $A \bullet B=e^{\log A+\log B}, A, B \in \mathcal{A}_{+}^{-1}$. 
Proof. We easily obtain that $I$ is the unit for the product $\bullet, \phi(I)=0$, and $\phi\left(A^{r}\right)=r \phi(A)$ holds for any $A \in \mathcal{A}_{+}^{-1}$ and rational number $r$. By the continuity of $\phi$ we obtain the boundedness of the map $P \mapsto \phi\left(e^{P}\right)$ on $\mathcal{P}(\mathcal{A})$ just as in the proof of Theorem 8. Clearly, this map is a bounded finitely additive $X$-valued measure on $\mathcal{P}(\mathcal{A})$, hence applying the solution of the Mackey-Gleason problem again, we deduce that there exists a bounded linear operator $L: \mathcal{A} \rightarrow X$ such that $\phi\left(e^{P}\right)=L(P), P \in \mathcal{P}(\mathcal{A})$. Again, as in the proof of Theorem 8, this implies that $\phi(A)=$ $L(\log A)$ holds for every $A \in \mathcal{A}_{+}^{-1}$. We infer

$$
\phi(A \bullet B)=\phi(A)+\phi(B)=L(\log A+\log B)=\phi\left(e^{\log A+\log B}\right), \quad A, B \in \mathcal{A}_{+}^{-1}
$$

from which we deduce by the injectivity of $\phi$ that

$$
A \bullet B=e^{\log A+\log B}, \quad A, B \in \mathcal{A}_{+}^{-1} .
$$

Remark 11. Apparently, it is a natural question that what happens in the type $\mathrm{I}_{2}$ case. The fact is that then the above conclusion in the statement fails to be valid. Indeed, to demonstrate it, as in [13, Remark 1], let $\psi$ be a continuous bijective map on the set all rank-one projections on a two-dimensional complex Hilbert space $H$ which does not preserve the transition probability (i.e., the trace of the product of rank-one projections) but does preserve orthogonality in both directions. Define the map $\tilde{\psi}$ on the space of all self-adjoint operators on $H$ by

$$
\tilde{\psi}(\lambda P+\mu Q)=\lambda \psi(P)+\mu \psi(Q)
$$

where $\lambda, \mu$ are real numbers and $P, Q$ are orthogonal rank-one projections. It can easily be seen that $\tilde{\psi}$ is a well-defined bijective map of the space of all self-adjoint operators on $H$. This map is not additive. Indeed, assume on the contrary that it is additive. Then by the continuity of $\tilde{\psi}$ we obtain that it is linear. Since it clearly maps projections to projections, we infer that it is a Jordan *-automorphism and hence it is implemented by a unitary or an antiunitary operator on $H$ (see, e.g., Theorems A.4., A.7 and A.8. in the appendix in [14]). This implies that $\psi$ preserves the transition probability, a contradiction.

Now define

$$
A \bullet B=\exp \left(\tilde{\psi}^{-1}(\tilde{\psi}(\log A)+\tilde{\psi}(\log B))\right), \quad A, B \in \mathcal{A}_{+}^{-1} .
$$

It requires elementary computations to verify that this operation $\bullet$ has the properties required in Theorem 10 (the injective continuous function $\phi$ being $\tilde{\psi} \circ \log$ ), but $\bullet$ is not the logarithmic product since $\tilde{\psi}$ is not additive.

Having read the material above one may be interested in the structure of the automorphisms with respect to the logarithmic product. But there is no relating structural result the reason for which is the following. The logarithmic product structure of a positive definite cone is clearly isomorphic to the usual additive group structure of the corresponding linear space of selfadjoint elements and, evidently, there is no particular structural result concerning the additive bijections of that space. However, the case is very much different for matrix algebras if we consider not only positive definite matrices but all positive semidefinite ones. The logarithmic product is extended for such matrices, too, using a certain limit process. The corresponding automorphisms have been completeley determined in [7].

\section{Concluding REMARKS}

We conclude the paper with some remarks, comments and open problems.

In Propositions 1, 2 we did not assume continuity while in Theorem 4 we did. It is a natural problem to investigate if the condition of continuity can be relaxed in the latter result. 
In the paper [20] we considered general symmetric means on positive semidefinite Hilbert space operators and determined their bijective preservers under a mild regularity assumption. It is also a natural question (we believe a difficult one) to consider the problem in the generality of the present paper, i.e., for positive definite cones in $C^{*}$-algebras or in von Neumann algebras.

As we have noted in Remark 9, there is no non-constant continuous map between the positive definite cones of von Neumann factors which would transform the geometric mean to the arithmetic mean. On the other hand, observe that the map $A \mapsto A^{-1}$ is a continuous bijective map on the positive definite cone of any $C^{*}$-algebra that transforms the harmonic mean to the arithmetic mean and vice versa. It seems an interesting question to clarify if there are other pairs of different (symmetric) means with the property that there do exist "isomorphisms" between them.

Less importantly, we note that several results in this paper could easily be extended to socalled weighted means. For the given parameter $t \in[0,1]$ the corresponding weighted arithmetic mean is

$$
(1-t) A+t B
$$

the weighted geometric mean is

$$
A \#_{t} B=A^{1 / 2}\left(A^{-1 / 2} B A^{-1 / 2}\right)^{t} A^{1 / 2},
$$

and the weighted harmonic mean is

$$
\left((1-t) A^{-1}+t B^{-1}\right)^{-1}
$$

Assuming $0<t<1$ (the cases $t=0$ or $t=1$ are trivialities) the so arising problems can in fact be reduced to the case where $t=1 / 2$ by applying simple iteration. This means that iterating the original given weighted mean in a proper manner and taking limit we arrive at the corresponding symmetric mean (the case where $t=1 / 2$ ). So, the considered preservers between given weighted means in the continuous case turn to be transformations between the corresponding symmetric means. Let us see how it works for the geometric mean which is the complicated one among those three operator means we are dealing with in this paper.

Let $t, 0<t<1$ be given and consider any other number $s \in[0,1]$. We show that the weighted geometric mean with the parameter $s$ can be obtained as the limit of an iteration process involving the weighted geometric mean with parameter $t$. Indeed, for a given pair $A, B$ of elements in $\mathcal{A}_{+}^{-1}$ we define the following sequences $\left(a_{n}\right),\left(b_{n}\right)$ of numbers (they do not depend on $A, B)$ and sequences $\left(A_{n}\right),\left(B_{n}\right)$ in $\mathcal{A}_{+}^{-1}$ recursively:

$$
\begin{aligned}
& a_{0}=0, b_{0}=1 A_{0}=A, B_{0}=B ; \\
& \text { if }(1-t) a_{n}+t b_{n} \leq s, \text { then set } a_{n+1}=(1-t) a_{n}+t b_{n}, b_{n+1}=b_{n} \text { and } A_{n+1}=A_{n} \#{ }_{t} B_{n}, \\
& B_{n+1}=B_{n} ; \\
& \text { if }(1-t) a_{n}+t b_{n}>s, \text { then set } a_{n+1}=a_{n}, b_{n+1}=(1-t) a_{n}+t b_{n} \text { and } A_{n+1}=A_{n}, \\
& B_{n+1}=A_{n} \#{ }_{t} B_{n} .
\end{aligned}
$$

It easily follows that $a_{n} \rightarrow s$ monotone increasingly and $b_{n} \rightarrow s$ monotone decreasingly. Moreover, it can be seen that

$$
A_{n}=A \#_{a_{n}} B, B_{n}=A \#_{b_{n}} B .
$$

Indeed, this follows from the following equality. For any real numbers $a, b$ from the unit interval we have

$$
\left(A \#_{a} B\right) \#_{t}\left(A \#_{b} B\right)=A \#(1-t) a+t b B .
$$

This equality can be checked by multipliying both sides by $A^{-1 / 2}$ and making use the transfer property. We have $A_{n}, B_{n} \rightarrow A \#_{s} B$, and both sequences $\left(A_{n}\right),\left(B_{n}\right)$ are obtained by iteration of $t$-weighted geometric means starting with $A, B$. 
So, if, for example, we have continuous maps $\phi: \mathcal{A}_{+}^{-1} \rightarrow \mathcal{B}_{+}^{-1}, \psi: \mathcal{A}_{+}^{-1} \rightarrow X$ such that

$$
\begin{gathered}
\phi\left(A \#_{t} B\right)=\phi(A) \#_{t} \phi(B), \quad A, B \in \mathcal{A}_{+}^{-1}, \\
\psi\left(A \#_{t} B\right)=(1-t) \psi(A)+t \psi(B), \quad A, B \in \mathcal{A}_{+}^{-1},
\end{gathered}
$$

then the same equalities hold for any given parameter $s \in[0,1]$ in the place of $t$, too. In that way one can easily extend Propositions 1, 2 and Theorems 4, 8 for weighted means.

We now make some algebraic comments. Consider $(A, B) \mapsto A \star B=(A \# B)^{2}$ as a new product on $\mathcal{A}_{+}^{-1}$. For commuting $A, B$ we have that it equals the usual product $A B$ in $\mathcal{A}$. We have seen above that the product $\star$ has the left alternative identity if and only if the underlying algebra $\mathcal{A}$ is commutative. Now, one may consider another natural product: $(A, B) \mapsto A * B=$ $A^{2} \# B^{2}$. It is easy to check that for any pair $A, B \in \mathcal{A}_{+}^{-1}$ we have

$$
(A * A) * B=A *(A * B)
$$

if and only if

$$
\left(A^{2} \star A^{2}\right) \star B^{2}=A^{2} \star\left(A^{2} \star B^{2}\right) .
$$

It follows that we have the left alternative identity for any one of the two products $\star, *$ exactly when we have it for the other. This implies that $\mathcal{A}_{+}^{-1}$ is a semigroup under the product $*$ if and only if $\mathcal{A}$ is commutative.

We continue as follows. Apparently, if there was a injective homomorphism from $\mathcal{A}_{+}^{-1}$ equipped with the product $\star$ to $\mathcal{B}_{+}^{-1}$ equipped with the logarithmic product, then the operation $\star$ would be associative which is the case only if the algebra $\mathcal{A}$ is commutative. In particular, we have that

$$
A \# B=\exp ((\log A+\log B) / 2)
$$

holds for all $A, B \in \mathcal{A}_{+}^{-1}$ if and only if $\mathcal{A}$ is commutative. (We recall that the expression on the right hand side of (9) is called the Log-Euclidean geometric mean of $A$ and $B$ but it is clearly not a mean in the Kubo-Ando sense.) We formulate a much stronger conjecture here. We believe that the validity of the above equality for any particular pair of $A, B$ (not for all pairs) implies that $A, B$ commute. We leave this as an open problem. Relating this question let us remark the following. Recall again that the standard K-loop product $\square$ on $\mathcal{A}_{+}^{-1}$ is defined by $A \boxminus B=A^{1 / 2} B A^{1 / 2}$. It is trivial to see that this operation always fulfills the left alternative identity. It was proved in [4, Proposition 3] that $\square$ on $\mathcal{A}_{+}^{-1}$ satisfies the right alternative identity or the flexible identity if and only if $\mathcal{A}$ is commutative. So, just as above it follows that if $\mathcal{A}_{+}^{-1}$ equipped with the product $\square$ can be embedded injectively into $\mathcal{B}_{+}^{-1}$ equipped with the logarithmic product, then $\mathcal{A}$ is commutative. In particular, if we have

$$
A^{1 / 2} B A^{1 / 2}=\exp (\log A+\log B), \quad A, B \in \mathcal{A}_{+}^{-1},
$$

then $\mathcal{A}$ is commutative. Let us consider the case of matrices. The well-known Golden-Thompson inequality states that

$$
\operatorname{Tr} e^{T+S} \leq \operatorname{Tr} e^{T} e^{S}
$$

holds for any pair $T, S$ of Hermitian matrices of the same size and in the inequality we have equality if and only if $T, S$ commute, see [26, Theorem 3.4]. Now, for positive definite complex matrices $A, B$ of the same size, if we have $A^{1 / 2} B A^{1 / 2}=\exp (\log A+\log B)$, then it implies

$$
\operatorname{Tr} e^{\log A} e^{\log B}=\operatorname{Tr}\left(e^{\log A}\right)^{1 / 2}\left(e^{\log B}\right)\left(e^{\log A}\right)^{1 / 2}=\operatorname{Tr} A^{1 / 2} B A^{1 / 2}=\operatorname{Tr} e^{\log A+\log B}
$$

which implies that $\log A, \log B$ commute and hence we obtain that $A, B$ commute. We do not know if (10) implies commutativity for positive definite Hilbert space operators, too, but we conjecture it does and, as already mentioned above, we have similar conjecture relating to the equality (9). One could continue proposing similar questions concerning other operations that appear in the paper, but we finish here. 


\section{REFERENCES}

1. T. Abe and O. Hatori, On a characterization of commutativity for $C^{*}$-algebras via gyrogroup operations, Period. Math. Hung., to appear.

2. M. Alexa, Linear combination of transformations, In SIGGRAPH'02: Proceedings of the 29th annual conference on computer graphics and interactive techniques (pp. 380-387), ACM Press, 2002.

3. W.N. Anderson and G.E. Trapp, Operator means and electrical networks, Proc. 1980 IEEE International Symposium on Circuits and Systems (1980), 523-527.

4. R. Beneduci and L. Molnár, On the standard K-loop structure of positive invertible elements in a $C^{*}$-algebra, J. Math. Anal. Appl. 420 (2014), 551-562.

5. R. Bhatia, Matrix Analysis, Graduate Texts in Mathematics, 169. Springer-Verlag, New York, 1997.

6. L.J. Bunce and D.M. Wright, The Mackey-Gleason problem, Bull. Amer. Math. Soc. 26 (1992), 288-293.

7. G. Dolinar and L. Molnár, Automorphisms for the logarithmic product of positive semidefinite operators, Linear Multilinear Algebra 61 (2013), 161-169.

8. R. Ger, On extensions of polynomial functions, Results Math. 26 (1994), 281-289.

9. O. Hatori and L. Molnár, Isometries of the unitary groups and Thompson isometries of the spaces of invertible positive elements in $C^{*}$-algebras, J. Math. Anal. Appl. 409 (2014), 158-167.

10. R.V. Kadison, A generalized Schwarz inequality and algebraic invariants for operator algebras, Ann. of Math. 56 (1952), 494-503.

11. R.V. Kadison and J.R. Ringrose, Fundamentals of the Theory of Operator Algebras, Vol II., Academic Press, 1986.

12. F. Kubo and T. Ando, Means of positive linear operators, Math. Ann. 246 (1980), 205-224.

13. L. Molnár and P. Šemrl, Non-linear commutativity preserving maps on self-adjoint operators, Quart. J. Math. 56 (2005), 589-595.

14. L. Molnár, Selected Preserver Problems on Algebraic Structures of Linear Operators and on Function Spaces, Lecture Notes in Mathematics, Vol. 1895, p. 236, Springer, 2007.

15. L. Molnár, Maps preserving the geometric mean of positive operators, Proc. Amer. Math. Soc. 137 (2009), $1763-1770$.

16. L. Molnár, Maps preserving the harmonic mean or the parallel sum of positive operators, Linear Algebra Appl. 430 (2009), 3058-3065.

17. L. Molnár, Thompson isometries of the space of invertible positive operators, Proc. Amer. Math. Soc. 137 (2009), 3849-3859.

18. L. Molnár, Order automorphisms on positive definite operators and a few applications, Linear Algebra Appl. 434 (2011), 2158-2169.

19. L. Molnár, Continuous maps on matrices transforming geometric mean to arithmetic mean, Annales Univ. Sci. Budapest., Sect. Comp. (special issue dedicated to Prof. Antal Járai on the occasion of his 60th birthday) 35 (2011), 217-222.

20. L. Molnár, Maps preserving general means of positive operators, Electron. J. Linear Algebra 22 (2011), 864-874.

21. L. Molnár, A few conditions for a $C^{*}$-algebra to be commutative, Abstr. Appl. Anal. Volume 2014 (2014), Article ID 705836, 4 pages.

22. L. Molnár, Jordan triple endomorphisms and isometries of spaces of positive definite matrices, Linear Multilinear Alg. 63 (2015), 12-33.

23. L. Molnár, General Mazur-Ulam type theorems and some applications, in Operator Semigroups Meet Complex Analysis, Harmonic Analysis and Mathematical Physics, W. Arendt, R. Chill, Y. Tomilov (Eds.), Operator Theory: Advances and Applications, Vol. 250, pp. 311-342, Birkhäuser, 2015.

24. L. Molnár, The logarithmic function and trace zero elements in finite von Neumann factors, Bull. Austral. Math. Soc., to appear.

25. L. Molnár and D. Virosztek, Continuous Jordan triple endomorphisms of $\mathbb{P}_{2}$, submitted. 
26. W. So, Equality cases in matrix exponential inequalities, SIAM J. Matrix Anal. Appl. 13 (1992), 1154-1158.

27. A. R. Sourour, Invertibility preserving linear maps on L(X), Trans. Amer. Math. Soc. 348 (1996) 13-30.

Department of Analysis, Bolyai Institute, University of Szeged, H-6720 Szeged, Aradi vértanúk tere 1., Hungary and MTA-DE "Lendület" Functional Analysis Research Group, Institute of Mathematics, University of Debrecen, H-4010 Debrecen, P.O. Box 12, Hungary

E-mail address: molnarl@math.u-szeged.hu

URL: http://www.math.u-szeged.hu/ molnarl/ 